\title{
A Multiscale Model for Quantitative Prediction of Insulin Aggregation Nucleation Kinetics
}

\author{
Rit Pratik Mishra and Gaurav Goel* \\ Department of Chemical Engineering, Indian Institute of Technology Delhi, Hauz Khas, \\ Delhi, 110016 (India) \\ E-mail: goelg@chemical.iitd.ac.in
}




\begin{abstract}
We combined kinetic, thermodynamic, and structural information from single molecule (protein folding) and two molecule (association) explicit-solvent simulations for determination of kinetic parameters in protein aggregation nucleation with insulin as model protein. A structural bioinformatics approach was developed to account for heterogeneity of aggregationprone species with the transition complex theory found applicable in modeling association kinetics involving non-native species. We show that a key simplification arises from presence of only a few relevant modes for non-native association kinetics. The kinetic parameters thus obtained were used in a population balance model and accurate predictions for aggregation nucleation time varying over two orders of magnitude with changes in concentration of insulin or an aggregation-inhibitor ligand were obtained while an empirical parameter set was not found to be transferable for prediction of ligand effects. This physically determined kinetic parameter set also allowed identification of the rate-limiting step in aggregation nucleation.
\end{abstract}

\title{
TOC Graphic
}

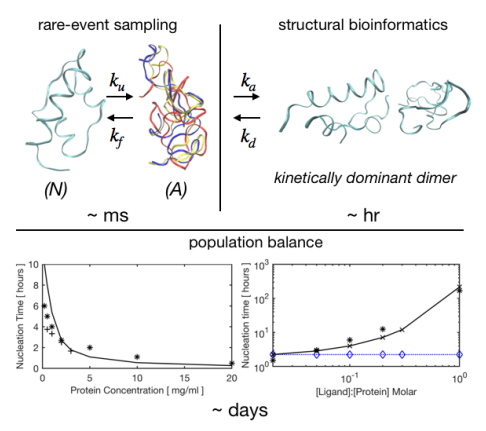


Protein drugs are a very important class of biotherapeutics with over ten thousands marketed products. Their in vivo or in vitro aggregation leads to issues such as loss of efficacy during storage ${ }^{1}$ or several severe disorders and diseases. ${ }^{2}$ A molecular level understanding of aggregation pathways can help in devising mitigation strategies. In particular, the knowledge of thermodynamics and kinetics of early stage aggregation nucleation events is necessary for developing strategies for inhibition of small oligomer formation that have been shown to be more cytotoxic than mature fibrils. ${ }^{3}$ However, their structural characterization ${ }^{45}$ and measurement of kinetic rate constants ${ }^{\sqrt[6]{8}}$ by bioanalytical techniques is made difficult by short life-time and a distinct, protein-dependent structure of species involved.The disparate timescales and lengthscales involved even for small proteins $(<12 \mathrm{kDa})$-milliseconds and nanometers for protein folding, seconds and tens of nanometers for aggregation nucleation, and hours-days and centimeters for fibrillation, preclude use of all-atom explicit solvent simulations for direct determination of aggregation thermodynamics and kinetics. Solvent-free, residue specific interaction models make accessible these timescales and lengthscales. ${ }^{910}$ However, these are biased towards formation of native interactions, and hence, not suitable for studying protein association leading to aggregation.

In another set of coarsegrained techniques, a protein is modeled as an isotropic or a patchy colloidal particle and protein association kinetics are then studied using either mesoscale simulations (Brownian dynamics, dissipative particle dynamics) or event-based methods (kinetic Monte Carlo simulations, population balance modeling $(\mathrm{PBM}))$. In several applications of these event-based approaches, the rates associated with elementary aggregation pathway steps are determined empirically to reproduce experimental measures of aggregation such as nuclei size, nucleation time, and growth kinetics. ${ }^{11}$ For example, PBM has been used to study the effect of osmolytes, protein concentration, stress factors like temperature, $\mathrm{pH}$, salt, impurities, and mechanical perturbation on protein aggregation.12 16 In this work, we present an approach for determination of a set of physically relevant kinetic parameters and show that a single parameter set is sufficient for accurate extrapolation of aggregation behaviour on variation of multiple factors. Insulin was used as a model protein because of its biotherapeutic relevance and availability of a large number of experimental studies on advancing understanding of its aggregation mechanism and kinetics. $17 / 18$

Table 1: Aggregation kinetics schemes. Scheme A: early events of aggregation that include protein folding-unfolding (rates: $k_{\mathrm{u}}, k_{\mathrm{f}}$ ) and sequential addition of an aggregation-prone monomeric species $\left(A_{1}\right.$, also referred to as $A$ for ease of notation) (rates: $\left.k_{\mathrm{nu}}, k_{-\mathrm{nu}}\right)$ to form higher order oligomeric species $\left(A_{i}\right)$ up to a stable nuclei of size $n_{c}^{*}$ (critical nucleus). Scheme B: same as Scheme A, but the protein association step explicitly represents diffusional association (rates: $k_{\mathrm{a}}, k_{\mathrm{d}}$ ) and conformational rearrangement (rates: $k_{\mathrm{r}}, k_{-\mathrm{r}}$ ). Scheme C: addition of ligand to aggregation-prone species smaller than the critical nucleus (rates: $k_{\mathrm{aL}}, k_{\mathrm{dL}}$ ). Scheme $\mathrm{D}$ : formation of higher-order fibrillar species and fibril fragmentation (rates: $k_{\mathrm{fib}}, k_{\mathrm{fib}-}, k_{\mathrm{frag}}$. For all schemes, $1 \leq i<n_{c}^{*}$. All species of size $\geq n_{c}^{*}$ are labelled $F$.

\begin{tabular}{|c|c|}
\hline Scheme A & $\begin{array}{c}\mathrm{N} \underset{k_{\mathrm{f}}}{\stackrel{k_{\mathrm{u}}}{\rightleftharpoons}} \mathrm{A}(1) \\
\mathrm{N}+\mathrm{A} \underset{k_{-\mathrm{nu}}}{\stackrel{k_{\mathrm{nu}}}{\rightleftharpoons}} \mathrm{A}_{2}(2) \\
\mathrm{A}_{i}+\mathrm{A} \underset{k_{-\mathrm{nu}}}{\stackrel{k_{\mathrm{nu}}}{\rightleftharpoons}} \mathrm{A}_{i+1}(3)\end{array}$ \\
\hline Scheme B & $\begin{array}{c}\mathrm{N} \underset{k_{\mathrm{f}}}{\stackrel{k_{\mathrm{u}}}{\rightleftharpoons}} \mathrm{A}(4) \\
\mathrm{N}+\mathrm{A} \underset{k_{\mathrm{d}}}{\stackrel{k_{\mathrm{a}}}{\rightleftharpoons}} \mathrm{N} \cdot \mathrm{A} \underset{k_{-\mathrm{r}}}{\stackrel{k_{\mathrm{r}}}{\rightleftharpoons}} \mathrm{A}_{2}(5) \\
\mathrm{A}_{i}+\mathrm{A} \underset{k_{\mathrm{d}}}{\stackrel{k_{\mathrm{a}}}{\rightleftharpoons}} \mathrm{A}_{i} \cdot \mathrm{A} \underset{k_{-\mathrm{r}}}{\stackrel{k_{\mathrm{r}}}{\rightleftharpoons}} \mathrm{A}_{i+1}(6)\end{array}$ \\
\hline Scheme C & $\mathrm{A}_{i}+\mathrm{L} \underset{k_{\mathrm{dL}}}{\stackrel{k_{\mathrm{aL}}}{\rightleftharpoons}} \mathrm{A}_{i} \cdot \mathrm{L}(7)$ \\
\hline Scheme D & $\begin{array}{l}\mathrm{A}_{\mathrm{i}}+\mathrm{F} \underset{k_{\mathrm{fb}-}}{\stackrel{k_{\mathrm{fb}}}{\longrightarrow}} \mathrm{F}(8) \\
\mathrm{F} \stackrel{k_{\mathrm{frag}}}{\longrightarrow} \mathrm{F}+\mathrm{F}(9)\end{array}$ \\
\hline
\end{tabular}

The extended Lumry-Eyring model, 19 used here to model aggregation kinetics, has been shown to be applicable to a large number of globular proteins ${ }^{11}$ including insulin. ${ }^{12[16}$ The overall model scheme, shown in Table 1, was divided in two parts: formation of a critical nucleus consisting of $n_{c}^{*}$ monomers by reversible 
addition of an aggregation-prone monomeric species $(A)$ to other monomers and small oligomers (Scheme A, equations 1 , 3) followed by an aggregation growth stage involving irreversible association and, possibly, conformational rearrangement of stable oligomers (size $\geq n_{c}^{*}$ ) to form fibrils (Scheme $\mathbf{D}$, equations 8 9). The first stage, referred to as early events of aggregation, is of interest in this work. It has been shown to have significant effect on aggregation rates and, in fact, is the ratedetermining step for many small proteins including insulin. $\frac{19}{19}$ In this case, the nucleation kinetics are determined by two key events: single molecule reconfiguration characterized by protein folding $\left(k_{\mathrm{f}}\right)$ and unfolding $\left(k_{\mathrm{u}}\right)$ rates, and dimer and higher oligomer formation characterized by protein-protein association $\left(k_{\mathrm{nu}}\right)$ and dissociation $\left(k_{-n u}\right)$ rates. These kinetic constants together determine the nucleation time $\left(t_{\mathrm{nu}}\right)$, defined as the induction time at which a measurable amount of stable oligomeric species have formed. An alternate scheme for aggregation nucleation that explicitly represents formation of a diffusional intermediate (involving a negligible change in conformation of associating species) followed by conformational rearrangement is shown in Scheme B (equations 46 ). A competing pathway involving aggregation inhibition by a ligand $(L)$ having a high affinity for aggregation-prone species $\left(A_{i}\right)$ is shown in Scheme C (equation 7). Role of both of these schemes in modulating aggregation kinetics and determination of kinetic constants appearing within are discussed later in this letter. We note that it is extremely important to account for the specificity in protein-protein and protein-ligand interactions involving nonnative aggregation-prone species and their effect on protein dimerization kinetics, the latter being a critical input to our model. To this end, we based our development on extensive explicit solvent simulations of a system consisting of either a single solute molecule (protein or ligand) or a single complex (protein dimer or protein-ligand complex) in-combination with a conformational selection model for protein association. 20121 Protein binding and folding in crowded environment, e.g. in cells, has been shown to occur at rates close to those determined in dilute solutions, $\stackrel{22}{2}$ implying that data obtained from simulations in the infinite dilution limit, i.e., only one (for protein folding) or two protein molecules (for protein dimerization) solvated in a large simulation box, will be relevant for modeling of aggregation kinetics.

Reduced Kinetic Model. Explicit solvent simulations to model protein association in large oligomer and fibrillar assemblies are intractable. We, therefore, investigated the effect of excluding fibrillation steps on species concentration and on estimation of the aggregation nucleation time $\left(t_{\mathrm{nu}}\right)$ of insulin. A critical nucleus size of $n_{c}^{*}=6$ was taken in accordance with earlier experimental and computational studies on insulin aggregation. ${ }^{12|16| 23 \mid 24}$ We calculated monomer $\left(C_{1}\right)$ and dimer $\left(C_{2}\right)$ concentrations (Figure 1) and the stable oligomer $\left(C_{i \geq 6} \equiv C_{\mathrm{F}}\right)$ concentration (Figure $3 \mathrm{~A}$ ) by solving either the full kinetic model (Scheme A and Scheme D) or only the early events kinetic model (Scheme A). The kinetic parameters have been taken from an earlier study wherein the full kinetic model was fitted to data on insulin aggregation growth. 12 The association $\left(k_{\text {nu }}\right)$ and dissociation $\left(k_{-n u}\right)$ rates for addition of species $A$ to any non-fibrillar species have been taken to be independent of oligomer size. The former can be explained on the basis of cancellation of the opposing effect on number of aggregation hotspots (increase with oligomer size) and oligomer rotational and translational diffusivity (decrease with oligomer size). The association rate for addition of $A$ to a pentameric oligomer is only two times larger than that for two monomeric species (Table S6, calculated using the Smoluchowski coagulation kernel ${ }^{25}$ ), and even more importantly, use of an oligomer-size dependent association rate does not affect calculation of nucleation time (Section S2.3). An oligomer size independent dissociation rate can be explained for a model where any binding interface consists of only two protein molecules. This is supported by observation of an increase in fibrillation rate on sonication that is expected to increase the number of fibril ends. ${ }^{26}$ Figure $3 \mathrm{~A}$ shows that $C_{\mathrm{F}}$ has a typical S-shaped temporal profile wherein there is a long induction time 


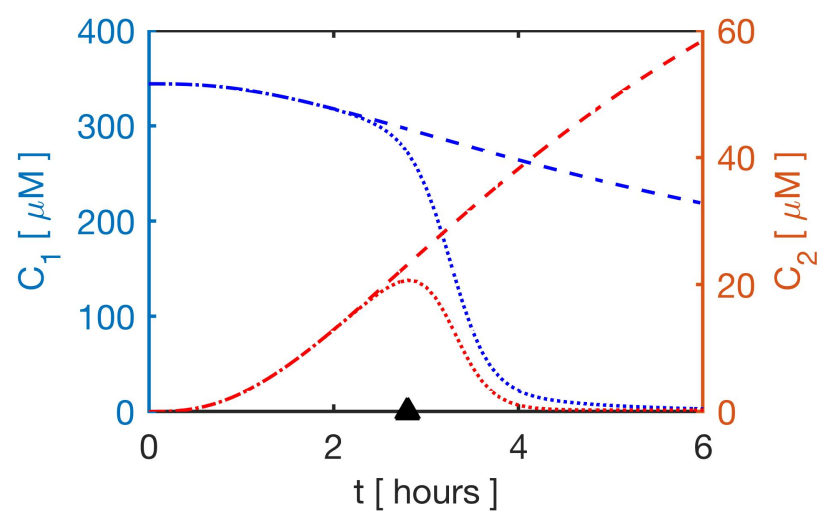

Figure 1: Reduced kinetic model for aggregation nucleation. Monomer $\left(C_{1}\right.$, in blue on left axis) and dimer $\left(C_{2}\right.$, in red on right axis) concentrations obtained by solving either the full kinetic model (..) or the reduced kinetic model (- -). Both models were solved using the kinetic parameters reported in Murray et al ${ }^{12}$ at $344 \mu \mathrm{M}$ insulin. The aggregation nucleation time $(\mathbf{\Lambda})$ is shown on time axis.

during which $C_{\mathrm{F}} \approx 0$. This induction time, defined as aggregation nucleation time $t_{\mathrm{nu}}$, was calculated as the time at which $C_{\mathrm{F}}$ is equal to $5 \%$ of its (long time) plateau value. The monomer and dimer concentrations obtained using the reduced kinetic model are in excellent agreement with the full model up to this nucleation time. The reduced kinetic model is clearly not appropriate for $t \geq t_{\mathrm{nu}}$, and therefore, $C_{\mathrm{F}}$ calculated using the reduced model does not have the S-shape profile, but still shows the presence of an induction time. In fact, the estimate of $t_{\mathrm{nu}}$ obtained from the two kinetic models are in excellent agreement, and this is not dependent on protein concentration and the method used to estimate the nucleation time (Section S2). This small effect of fibrillation can be explained on basis of very small fibril concentration till the nucleation time. Therefore, we conclude that the reduced kinetic model can be used to accurately determine the aggregation nucleation time, and the monomer and dimer concentration up to $t_{\text {nu }}$. This forms a very important basis for our approach to estimate physically correct values for various rate constants in the kinetic model.

Diffusional Association Rates. Under the assumptions made here, the reduced kinetic model is fully characterized by thermodynamics and kinetics of two events: (i) protein foldingunfolding and (ii) protein dimerization involving at least one aggregation prone species $(A)$. We have taken insulin folding $\left(k_{\mathrm{f}}\right)$ and unfolding $\left(k_{\mathrm{u}}\right)$ rates from our previous study where metadynamics simulations were used to characterize the insulin folding landscape. ${ }^{27}$ Further, three metastable basins, each consisting of kinetically connected partially-folded conformations (collectively labeled as $A$ here), in addition to a native $(N)$ and a denatured $(D)$ basin were identified in that study. A first-principles treatment of addition of $A$ to a monomer $(N$ or $A$ ) or a sub-nuclei oligomer $\left(A_{i}, 2 \leq i \leq 5\right)$, as given in Table 1, requires the determination of rate constants $\left(k_{\mathrm{a}}, k_{\mathrm{d}}\right)$ for reversible diffusional association of the N.A complex. For the association rate $k_{\mathrm{a}}$, we have used the transition complex theory that has been shown to provide accurate predictions for kinetics of diffusional association of several protein-receptor pairs in their native state. ${ }^{28 \mid 29}$ Specifically, it was shown to suitably account for the effect of long-range electrostatic interactions on the so-called basal association rate, the latter determined solely from unbiased translation and rotation of a pair of rigid-bodies to form native protein-protein contacts. This is important in the present case since there is a net charge of $+7 e$ (at $\mathrm{pH} 2)$ on the N.A dimer. Given the structural heterogeneity of partially-folded species $A$ and lack of knowledge on the structure of transient $N$.A dimer, we first determined an ensemble of representative structures for the N.A dimer to be used as input to the transition complex theory.

Structure of N.A dimer(s) in optimal binding mode. A set of 16 most stable (lowest binding free energy) N.A insulin dimer conformations were taken from our earlier study wherein extensive rigid-body docking was used to generate a large ensemble of possible dimer conformations, followed by conformation ranking on basis of binding free energies calculated using the molecular mechanics Poisson-Boltzmann surface area (MM-PBSA) implicit solvent model. $\underline{30}$ We carried out an extensive set of molecular dynamics simulations starting with these 16 structures with a specific aim of allowing reorientation of binding partners under influence 
of long-range electrostatics and refinement of contacts determined from rigid-body docking. To allow for the determination of an electrostatically optimal binding mode, five replicate simulations were done for each rigid-body docked structure wherein monomer $A$ in any given N.A dimer was moved perpendicular to the binding interface by a distance equal to twice the Debye length $\left(2 \lambda_{\mathrm{D}}=2.2 \mathrm{~nm}\right.$ at $\left.100 \mathrm{mM} \mathrm{NaCl}\right)$ to ensure a negligible effect of any specific proteinprotein interactions (PPIs) at this initial separation (see Figure S1). Since at this step we are only interested in capturing the effect of long-range electrostatic interactions between the $N$ and $A$ monomers screened by the aqueous buffer, we have used the coarsegrained (CG) MARTINI forcefield with the polarizable water model. ${ }^{31}$ This was shown to accurately reproduce screening effects in aqueous solutions, and therefore, provides best compromise between speed and accuracy: all-atomistic simulations spanning several microseconds for a system with over one million atoms are intractable while implicit solvent forcefields (for example, Gō-like models) will not correctly reproduce screened electrostatic interactions. Each CG simulation was run for $300 \mathrm{~ns}$, giving a total of $24 \mu$ s simulation time.

41 out of 80 CG-MARTINI simulations resulted in re-formation of the N.A dimer for an extended time during the $300 \mathrm{~ns}$ simulation. In all cases where a complex was formed, it was found to have a stable conformation for at least last $100 \mathrm{~ns}$ of the simulation (Figure S3). However, the binding interface area for the final structure obtained after CG-MARTINI simulation was found to be lower than that for the rigid-body docked dimer (Table S2), possibly resulting from a larger bead size in the $\mathrm{CG}$ model. We therefore carried out a $20 \mathrm{~ns}$ allatomistic (AA) simulation for each of the 41 re-formed complexes, with the initial structure taken as the central structure of the highest population cluster in the stable part of the $\mathrm{CG}$ simulation. The binding interface area quickly increased during the AA simulation to a value very close (in most cases greater) to that in the rigid-body docked dimers (Table $\mathrm{S} 2$ and S3) and, thereafter, remained stable (data not shown). For each of the 41 N.A dimers obtained at the end of AA simulations, the highest binding interface similarity score (equation S13) with any one of the 16 rigid-body docked complexes lies between 0.39-0.74 (maximum possible similarity score value is 1 ), thus indicating a refinement in dimer structure rather than a complete change. In summary, the combination of CG-MARTINI and AA simulation allowed us to obtain a set of optimized dimer structures, with the former allowing us to achieve a better electrostatic complementarity at a manageable computational cost and the latter providing for better packing at the binding interface.

Kinetically Dominant Binding Mode(s). From the large set of binding modes for formation of a N.A dimer, we expect only a small subset to be relevant for aggregation nucleation. To find this subset of important binding modes, we first group kinetically similar dimer structures together using a reaction criterion employed in Brownian dynamics simulation schemes for diffusional protein-protein association: a successful encounter complex was considered to be formed when two independent binding interface contacts were formed simultaneously. ${ }^{32}$ We obtained eight clusters on applying this criterion in-combination with integer linear programming to minimize the number of clusters (details in Section S1.2). Each cluster (largest: 16 structures, smallest: 2 structures), consisting of structures with a distinct set of binding interface residues is representative of a distinctive binding mode (full list of cluster sizes and binding modes in Figure S4).

We solved the subset of rate equations in Scheme B up to the formation of the N.A complex to determine which binding modes dominate the formation of this diffusional dimer. The calculation of diffusional association rate $\left(k_{\mathrm{a}}\right)$ using the transition complex theory requires the existence of a transition state separating the bound and the unbound state of a complex where no large-scale conformational changes have occurred. Figure 2A shows that indeed the transition state of N.A dimer is located at the outer boundary of the bound state, the latter defined by a deep interaction energy well. As done in the original work, the to- 

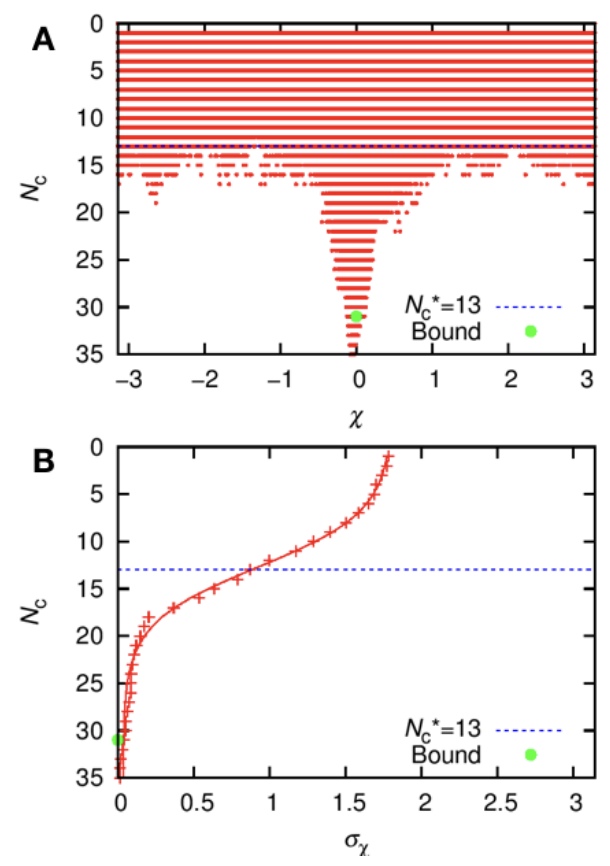

Figure 2: Energy landscape for $N . A$ complex. (A) Scatter plot for total number of contacts, $N_{C}$, in the ensemble of structures obtained on a rotation $\chi$ away from the bound complex. (B) Standard deviation of the rotational angle, $\sigma_{\chi}$, plotted versus $N_{C}$. The data is shown for the kinetically dominant $N$.A dimer. The total number of contacts in the bound state and in the transition state was 32 and 13, respectively. Plots generated using TransComp. 33

tal number of contacts in any configuration, $N_{\text {c }}$, has been used as a measure of interaction energy. Figure 2B shows a sharp increase in standard deviation of the rotational angle $(\chi), \sigma_{\chi}$, at the contact level of the transition state $\left(=N_{\mathrm{c}}^{*}\right)$, indicative of a large increase in rotational freedom on reaching the transition state. In fact, $N_{\mathrm{c}}^{*}$ was determined from an order parameter based on the distribution $\sigma_{\chi}\left(N_{\mathrm{c}}\right)$. For several associating protein pairs with unlike charges, it was shown that the transition state involved the formation of an electrostatically complementary conformation that lead to an enhancement of protein association rates compared with the basal association rate. ${ }^{28132}$ Table 2 shows that indeed $\left(k_{\mathrm{a}}\right)$ for various $N . A$ insulin dimers is strongly correlated with the electrostatic interaction energy of the transition state complex, $\Delta G_{N . A}^{* \text { (elec) }}$, whereas only weakly correlated with the binding free energy of the bound complex, $\Delta G_{N . A}^{\mathrm{o}}$. The Pearson correlation coef-
Table 2: Kinetic and thermodynamic parameters for eight binding modes of N.A dimer. The association rate of N.A dimer, $k_{\mathrm{a}}$, and the electrostatic interaction energy of the corresponding transition state complex, $\Delta G_{N . A}^{*(\text { elec })}$, were determined using the transition complex theory. The binding free energy of the N.A dimer, $\Delta G_{N . A}^{\mathrm{o}}$, was calculated using the MM-PBSA method on a 20 ns MD trajectory. It also includes the contribution from protein configurational entropy change estimated using an atom specific weighted surface area (WSAS) method. 34

\begin{tabular}{cccc}
\hline \hline$N A^{i}$ & $\begin{array}{c}k_{\mathrm{a}}^{i} \\
\left(\mu \mathrm{M}^{-1} \mathrm{hr}^{-1}\right)\end{array}$ & $\begin{array}{c}\Delta G_{N . A}^{*(\mathrm{elec})} \\
\mathrm{kcal} \mathrm{mol}^{-1}\end{array}$ & $\begin{array}{c}\Delta G_{N . A}^{\mathrm{o}} \\
\mathrm{kcal} \mathrm{mol}^{-1}\end{array}$ \\
\hline 1 & 6734 & 0.694 & -3.84 \\
2 & 1332 & 0.937 & -1.97 \\
3 & 536 & 1.237 & -2.99 \\
4 & 376 & 1.184 & 0.234 \\
5 & 350 & 1.498 & -2.85 \\
6 & 147.2 & 3.221 & 1.2 \\
7 & 12.08 & 5.123 & 0.64 \\
8 & 4.06 & 1.781 & -2.24 \\
\hline \hline
\end{tabular}

ficient for top five binding modes (as per $\left(k_{\mathrm{a}}\right)$ ) was obtained to be -0.83 for the former and -0.57 for the latter. In spite of the same sign of net charge on $N(+4 e)$ and $A(+3 e)$ monomers, $\Delta G_{\text {elec }}$ has a small positive value $(\sim$ thermal en$\left.\operatorname{ergy}\left(=1.5 R T=1 \mathrm{kcal} \mathrm{mol}^{-1}\right)\right)$ for dimers with four highest $\left(k_{\mathrm{a}}\right)$. Overall, we have shown that the key requirements concerning the nature of the transition complex postulated in the theory by Zhou and co-workers, so-far used only for modeling native protein-protein association, are satisfied in the present case for non-native association leading to aggregation nucleation. The estimation of the dissociation rate, $k_{\mathrm{d}}$, from the binding free energy of $N . A$ dimer, $\Delta G_{N . A}^{\mathrm{o}}$, provided us the full set of kinetic parameters (given in Table 2) required to obtain the dimer concentration in each binding mode.

For each of the eight binding modes, the structure with the lowest (most negative) $\Delta G_{N . A}^{o}$ (preferable over $\Delta G_{N . A}^{*(\text { elec })}$ since it is correlated with both $k_{\mathrm{a}}$ and $k_{\mathrm{d}}$ ) was used for the determination of temporal evolution of the concentration of $N . A$ dimer $([N . A])$ in that mode. Table 2 shows there is significant variation in $k_{\mathrm{a}}$ and $\Delta G_{N . A}^{\mathrm{o}}$ for these eight structures. 
The $k_{\mathrm{a}}$ values of order $10^{3} \mathrm{\mu M} \mathrm{h}^{-1}$ obtained here are typical for diffusional association of protein complexes (e.g., see Table 3), but are seven orders of magnitude larger that the overall association rate, $k_{\mathrm{nu}}$, for the formation of the final dimeric complex, $A_{2}$ (Table 3). This clearly indicates that insulin dimerization is not diffusion-limited and must involve a slow conformational rearrangement step, as given by equations 5 and 6 in Scheme B. We discuss one important implication of this revised kinetic scheme in the later part of this letter where it is shown that inclusion of conformational rearrangement is necessary for quantification of effect of ligands on insulin aggregation nucleation time. Figure $\mathrm{S} 5 \mathrm{~A}$ shows that one particular binding mode (binding mode 1 in Table 2) has a significantly higher value for $[N . A]$ compared to any other mode. We acknowledge that this observation of a single dominant binding mode analogous to native protein-receptor binding might be fortuitous resulting from approximations involved in calculation of binding energy using the MM-PBSA-WSAS approach. A direct validation of $\Delta G_{N . A}^{o}$ estimated here is not possible as corresponding experimental estimation of equilibrium data for non-native insulin dimerization is made difficult by presence of several higher-order oligomers and fibrils. However, the value obtained $\left(-3.84 \mathrm{kcal} \mathrm{mol}^{-1}\right.$ for the most stable binding mode in Table 2 is comparable to that for native insulin dimers, which is the dominant species at acidic $\mathrm{pH} .17$ For example, the binding free energy for dimerization of porcine insulin (different from human insulin only at amino acid 30 of $\mathrm{B}$ chain: TB30A) at $298 \mathrm{~K}$ and $\mathrm{pH} 2$, determined using concentration difference spectroscopy, was reported to be $-6.8 \mathrm{kcal} \mathrm{mol}^{-1} \underline{35}$ The presence of specificity in non-native interactions can also be related to the observation that the partiallyfolded, aggregation-prone conformations of insulin and many other proteins are known to have a distinct structure with residual native contacts. $36 \mid 37$ Therefore, we expect to see similarities in characteristics of protein association involving such PFIs and that involving only native proteins, as observed also in applicability of the transition complex theory to the N.A
Table 3: Kinetic parameters in insulin aggregation nucleation. Column A : Present work; Column B : Full set of parameters determined empirically by fitting to aggregation growth data; ${ }^{12}$ Column C : direct experimental measurements for individual elementary steps for insulin or other globular proteins.

\begin{tabular}{l|lll}
\hline $\begin{array}{l}\text { Rate } \\
\text { constant }\end{array}$ & A & B & C \\
\hline$k_{n u}$, & $1.59 \times 10^{-3} 1.8 \times 10^{-4}$ & $\left(10^{-3}-\right.$ \\
$\mu \mathrm{M}^{-1} \mathrm{~h}^{-1}$ & & & $\left.10^{-4}\right)^{\underline{41}}$ \\
$K_{A_{2}}, \mu M$ & 22 & 45 & $12^{\sqrt[35 / 42]{42}}$ \\
$k_{a}$ & 6.74 & - & $\left(10^{3}-\right.$ \\
$K_{N . A}, \mu M$ & $\times 10^{3}$ & & $\left.10^{6}\right)^{28|43| 44}$ \\
$f$ & $3.3 \times 10^{3}$ & - & - \\
$k_{u}, \mathrm{~h}^{-1}$ & .07 & .99 & $(0-$ \\
$k_{f}, \mathrm{~h}^{-1}$ & $2.4 \times 10^{8}$ & 0.81 & $\left(10^{5}-\right.$ \\
$k_{r}, \mathrm{~h}^{-1}$ & $2.8 \times 10^{9}$ & $3.6 \times 10^{-3}$ & $\left.10^{11}\right)^{\underline{45 \mid 46}}$ \\
\hline
\end{tabular}

dimer. We, therefore, conjecture that only a small number of binding modes, even if not only one mode as determined here, will be relevant for insulin aggregation nucleation and subsequent fibrillation. The presence of structural specificity in protein interactions involved in insulin aggregation is also supported indirectly by some experimental data, such as the proposal that fibril growth occurs by addition of an insulin PFI only to the ends of an existing fibril, $\underline{38}$ significant inhibition of insulin aggregation at equimolar or smaller concentrations of a ligand with high affinity for insulin PFIs. ${ }^{39140}$ The identification of dominant binding modes has allowed us to determine the hotspot residues and dominant interactions in non-native association of insulin (Figure S5B) that can in-turn be used as input for high-throughput screening of ligands for disrupting protein-protein interactions leading to insulin aggregation.

Role of Dimer Conformational Rearrangement. We have noted above a significant difference in diffusional association rate for $N . A$ dimer $\left(k_{\mathrm{a}} \sim 10^{3} \mathrm{\mu M} \mathrm{h}^{-1}\right)$ and the estimate for association rate of final dimeric species $A_{2}$ $\left(k_{\mathrm{nu}} \sim 10^{-4} \mu \mathrm{Mh}^{-1}\right)$. This implies that N.A 
and $A_{2}$ must be separated by a slow dimer conformational rearrangement step as shown in Scheme B(equations 4 6), with $k_{\mathrm{r}}$ representing the forward rate for conformational change of $N . A$ to $A_{2}$. Since $k_{\mathrm{nu}}<<k_{\mathrm{a}}$, equation $\mathrm{S} 26$ simplifies to $k_{\mathrm{r}} \approx k_{\mathrm{nu}} \exp \frac{\Delta G_{N . A}^{o}}{R T}$, implying that a given overall dimerization rate, $\left(k_{\text {nu }}\right)$, can be obtained for infinite combinations of $k_{\mathrm{r}}$ and $\Delta G_{N . A}^{\mathrm{o}}$. We have taken $k_{\mathrm{r}}$ as an adjustable parameter to be determined from data on aggregation growth while $\Delta G_{N . A}^{\mathrm{o}}$ was calculated using the MM-PBSA-WSAS method as explained above. In fact, $k_{\mathrm{r}}$ is the only adjustable parameter in our reduced kinetic model. All other kinetic parameters, reported in Table 3, were fixed at values obtained by our first-principles approach. The binding free energy of the aggregation pathway dimeric species $\left(A_{2}\right)$ is expected to be different from $\Delta G_{N . A}^{\mathrm{o}}$ (because of dimer conformational re-arrangement) and was taken to be equal to the experimental estimate available for binding free energy of native insulin dimerization (dimer equilibrium dissociation constant $K_{A_{2}}=12 \mu \mathrm{M}$ corresponding to $\left.\Delta G_{A_{2}}^{\mathrm{o}}=-6.8 \mathrm{kcal} \mathrm{mol}^{-1}\right) . k_{\mathrm{r}}$ was then estimated by solving the resulting reduced kinetic model Scheme B for time variation of total monomer concentration, $C_{1}(t)$, and performing a least-squares fit to $C_{1}(t)$ obtained from the full kinetic model without conformational rearrangement (Schemes A, D). The parameters for the full kinetic model were taken from an earlier study that showed excellent agreement with experimental data on fibril growth kinetics. ${ }^{[12}$ We have used the data only up to the nucleation time $\left(t_{\mathrm{nu}}=2.8 \mathrm{~h}\right.$ at $344 \mu \mathrm{M}$ insulin) in-accordance with the validity of reduced kinetic model and obtained $k_{\mathrm{r}}=5.2 \mathrm{~h}^{-1}$. A simulated annealing protocol with several distinct initial guesses for $k_{\mathrm{r}}$ gave the same solution in each case. Even when $K_{A_{2}}$ was also taken as an unknown parameter, we obtained $k_{\mathrm{r}}=5.05 \mathrm{~h}^{-1}$, essentially the same as previous estimate. In this case, we obtained $K_{A_{2}}=22 \mu \mathrm{M}$, very close to the fixed value of $K_{A_{2}}=12 \mu \mathrm{M}$. This implies that $k_{\mathrm{r}}=5.2 \mathrm{~h}^{-1}$ is a robust estimate for rearrangement rate and is several orders of magnitude smaller than effective diffusional association rate $\left(k_{\mathrm{a}} C_{1}(0)=2.3 \times 10^{6} \mathrm{~h}^{-1}\right)$. Slow rear- rangement of pre-fibrillar aggregates has been reported for insulin $\underline{48}$ and other small globular proteins, $\frac{47 \mid 4950}{15}$ with rates several orders of magnitude smaller than those corresponding to protein folding or $\beta$-sheet formation at interface of two small peptides. The proposal that this much smaller rearrangement rate compared to intra- and inter-protein $\beta$-sheet formation can be explained on basis of double layer repulsion and reconfiguration ${ }^{51}$ is not supported by the observations in our study. For example, for an initial separation of more than two Debye lengths between two associating insulin monomers ( $N$ and $A$ ) ensuring almost complete formation of a ionic double layer with a total protein charge of $+7 e, 50 \%$ of stable dimer complexes (total 80 simulations) were formed in less than $100 \mathrm{~ns}$ of simulation time. An alternate mechanism, based on characterization of early events of insulin aggregation using orthogonal bioanalytical techniques, proposes a slow, sequential rearrangement of prefibrillar oligomeric species after diffusional association. $\frac{48152}{}$ This slow rearrangement of prefibrillar aggregates on a timescale of more than $10 \mathrm{~h}$ has also been reported for other amyloid proteins such as $A \beta 40$. $\underline{4749 / 50}$

Using the estimate for $k_{\mathrm{r}}$ in equation S26. we get the overall dimer association rate $k_{\mathrm{nu}}=$ $1.6 \times 10^{-3} \mathrm{\mu M}^{-1} \mathrm{~h}^{-1}$, which is an order of magnitude larger than the value obtained when all kinetic rate constants were treated as adjustable parameters and determined simultaneously using least-squares fitting $\frac{12}{12}$ (see Table 3). This difference in $k_{\mathrm{nu}}$ is offset by the difference in the native monomer fraction for foldingunfolding equilibria: we have used a value of $f=0.07$ as obtained from metadynamics simulations $^{27}$ in contrast to $f=0.99$ obtained by least-squares fitting. 12 Insulin formulations incubated at aggregation-prone conditions of acidic $\mathrm{pH}(2-3)$ and high temperature $\left(40{ }^{\circ} \mathrm{C}-\right.$ $\left.60^{\circ} \mathrm{C}\right)$, but low concentration $(\approx 100 \mathrm{nM})$, had the native protein as dominant species as determined using Fourier transform infrared (FTIR) spectroscopy with $f=0-0.15 . \underline{53 \mid 54}$ Therefore, simultaneous estimation of multiple kinetic parameters by fitting to experimental data on growth kinetics can yield estimates significantly 
different from values obtained by direct quantitative methods.
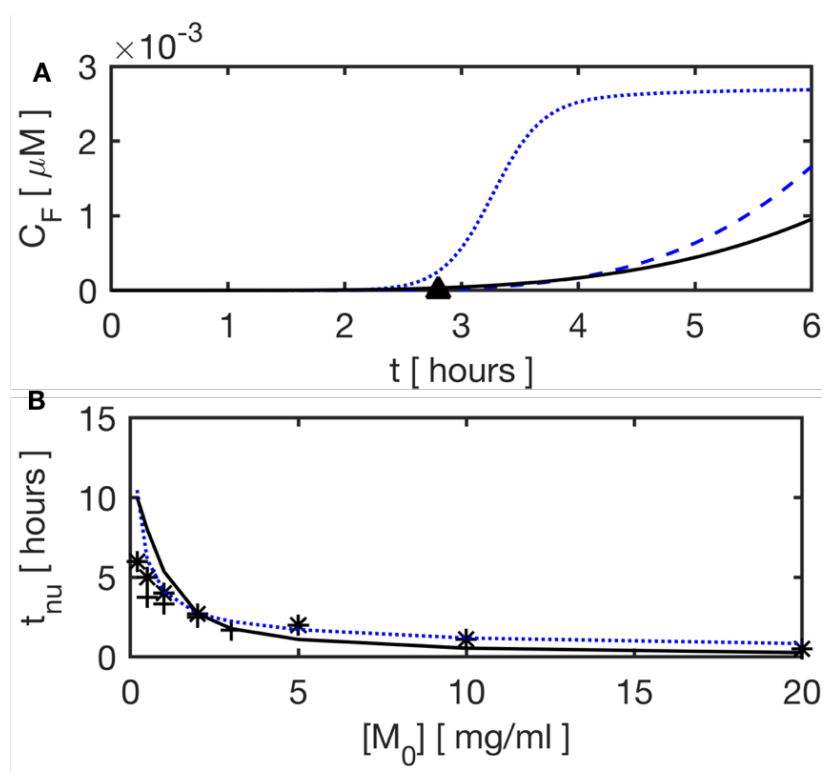

Figure 3: Effect of protein (insulin) concentration on aggregation kinetics. (A) Temporal evolution of fibril concentration, $C_{\mathrm{F}}$, obtained using the full kinetic model $(. \cdot)$, and the reduced kinetic models either excluding ( - - ) or including ( - ) dimer conformational rearrangement. (B) Aggregation nucleation time $\left(t_{\mathrm{nu}}\right)$ as function of initial insulin concentration $\left(\left[M_{0}\right]\right)$ obtained from kinetic models compared with the corresponding experimental estimate $((*)$ Lee et al. $.16,(+)$ Amdursky et al. $\left.{ }^{55}\right)$.

\section{Prediction of Effect of Protein and Ligand} Concentration on Aggregation Nucleation. Figure 3 A shows the comparison of prediction for stable oligomer concentration $\left(C_{i \geq 6} \equiv C_{\mathrm{F}}\right)$ obtained by solving the full kinetic model (parameters from ref. ${ }^{12}$ ), and the reduced kinetic model without rearrangement (parameters from ref. ${ }^{12}$ ) or with rearrangement (present work, parameters in Table 3). All three cases show almost equal induction time. However, as discussed above, the reduced model does not have an inflection point in $C_{\mathrm{F}}(t)$, and therefore, can not be used to directly determine the aggregation nucleation time, $t_{\mathrm{nu}}$. Instead, we used the relative fibril concentration at $t_{\mathrm{nu}}$, defined for a given initial protein concentration $\left[M_{0}\right]$ as $\kappa_{\mathrm{n}}=\frac{C_{\mathrm{F}}\left(t_{\mathrm{nu}}\right)}{\left[M_{0}\right]}$, calculated at $344 \mu \mathrm{M}$ insulin as a fixed parameter to determine the nucleation time at all other state points(Section S2.2 for details). Even when $\kappa_{\mathrm{n}}$ was estimated indepen- dently at each $\left[M_{0}\right]$, it stays essentially constant for large changes in $\left[M_{0}\right]\left((2-20) \mathrm{mg} \mathrm{ml}^{-1}\right)$ (Table S5). The monomer and dimer concentrations obtained using either of the two reduced models are in excellent agreement with the full model up to $t_{\mathrm{nu}}$ (Figure S9), implying that both reduced models can be used to obtain estimates for aggregation nucleation time.

We first compare predictions from different kinetic models and parameter estimation methods against the data available on effect of initial insulin concentration on aggregation nucleation time. We have kept all model parameters, estimated either by fitting to aggregation growth data at $344 \mu \mathrm{M}\left(1.97 \mathrm{mg} \mathrm{ml}^{-1}\right)$ insulin $\left(k_{\mathrm{r}}, \kappa_{\mathrm{n}}\right)$ or by direct determination for the elementary step under consideration $\left(k_{\mathrm{u}}, k_{\mathrm{f}}, k_{\mathrm{a}}, K_{N . A}, K_{A_{2}}\right)$, fixed at all protein and ligand concentrations (given in Table 3). Figure $3 \mathrm{~B}$ shows that estimate for $t_{\mathrm{nu}}$ obtained using these physically determined parameter values in the reduced kinetic model are in excellent agreement with corresponding values determined from a diverse set of bioanalytical techniques for initial insulin concentrations in range $(0.1$ to 20$) \mathrm{mg} \mathrm{ml}^{-116 \mid 55}$ leading to a variation of over two orders in $t_{\mathrm{nu}}$. The full kinetic model, using parameters determined by least-squares fit to aggregation growth data at $344 \mu \mathrm{M}, \stackrel{12}{12}$ provides predictions for $t_{\mathrm{nu}}$ at the same accuracy level. In both cases, a higher estimate (compared to experimental data) for $t_{\text {nu }}$ at low concentrations could be explained by increased importance of heterogeneous nucleation (from contained walls, solution-air interface) at low concentrations that has not been included in our kinetic scheme.

We observe that large differences in kinetic parameter values obtained using direct determination at the level of elementary step versus the case when full set is determined empirically (see Table 3) does not affect the quality of prediction of aggregation kinetics variation with protein concentration. This can be attributed to absence of any competing reaction steps in the kinetic schemes when investigating role of protein concentration, thereby making possible offsetting of deviations in model parameter values. For example, a significantly larger value for 


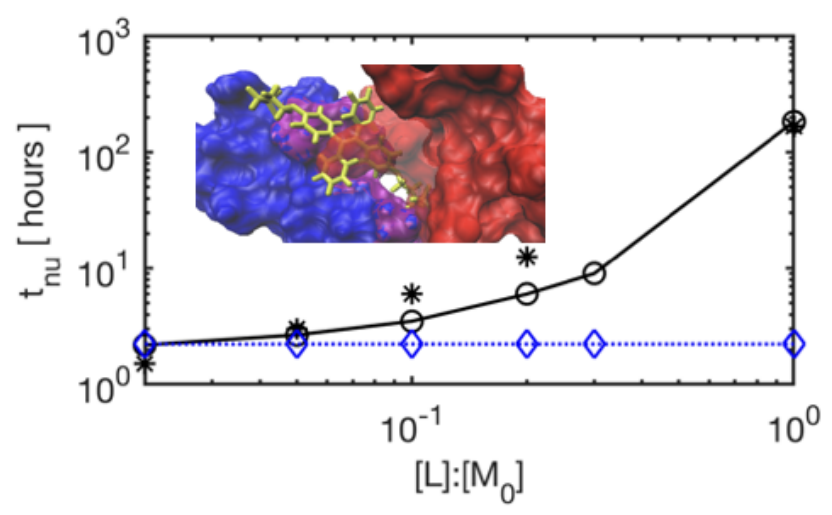

Figure 4: Effect of ligand (BSPOTPE) concentration on aggregation kinetics. Aggregation nucleation time $\left(t_{\mathrm{nu}}\right)$ as function of BSPOTPE concentration $(L)$ at $500 \mu \mathrm{M}$ insulin $\left(\left[M_{0}\right]\right)$ obtained by solving either the full model with empirical parameter set from ref. $12\left(-\nabla_{-}\right)$or the reduced model with parameters determined in this work $(-\circ-)$. In both cases, ligand inhibition was modeled using equation 7. Experimental estimate from Hong et al ${ }^{\frac{39}{39}}$ is also shown $(*)$. Inset: A snapshot of the overlay of kinetically dominant $N . A$ complex and the $A . L$ complex zoomed-in at the binding interface $(N$ : red (surf view), $A$ : blue (surf view), $L$ : green (bonds view).

the equilibrium fraction of aggregation-prone monomer was offset by a reduced dimerization rate in the empirically determined set. A more stringent test is provided by cases where conditions are changed to affect a particular elementary step (for instance, a sequence mutation that affects protein stability), affect various elementary steps differently (for instance, change in salt concentration will have different effect on folding equilibria and association equilibria), or when a competing reaction is added to the kinetic model. Here we have taken up the last case, wherein a ligand with specific affinity for the aggregation-prone species $(A)$ was added to the protein formulation.

We have used the data available on inhibition of insulin aggregation by 1,2 -Bis [4-(3sulfonatopropoxyl)phenyl]-1,2-diphenylethene salt (BSPOTPE), an organic fluorogen that was postulated to bind predominantly at hydrophobic sites on the aggregation-prone monomeric and oligomeric species. ${ }^{39}$ In it the photo luminescence (PL) spectrum was used to determine aggregation nucleation time for insulin incubated with BSPOTPE at $330 \mathrm{~K}$ and $\mathrm{pH}$
2. The additional kinetic equations to account for ligand $(L)$ effect are given by Scheme C (equation 7). For calculation of kinetic parameters, several putative structures for the A.L complex were determined by separately docking five distinct conformations of BSPOTPE at each of the ten dimerization hotspot residues of partially folded species $A$. The top docked structures were found to be metastable with the binding interface staying essentially the same over a $20 \mathrm{~ns}$ MD simulation (for example see Figure $\mathrm{S} 6 \mathrm{~A}$ and $\mathrm{S} 6 \mathrm{~B})$. The binding interface area of species $A$ in the most stable $N$.A complex $\left(A_{i}^{\mathrm{N} . \mathrm{A}}\right)$ and in the most stable $A . L$ complex $\left(A_{i}^{\text {A.L }}\right)$, shown in Figure 4 (inset), was $4.1 \mathrm{~nm}^{2}$ and $2.8 \mathrm{~nm}^{2}$, respectively. This implies that only one BSPOTPE molecule can bind to the hotspot residues of $A$ in the N.A complex, and accordingly, our reaction scheme involved only one ligand binding event. The $A . L$ structure with highest $\cos \Theta^{A . L, N . A}$ (defined in equation S13), a parameter calculated from A.L binding energy and similarity of binding interface residues in this complex to insulin dimerization hotspot residues, was used for the calculation of ligand association rate from the transition complex theory (see Methods Section S1.7 for details). We obtained $k_{\mathrm{aL}}=9.23 \times 10^{4} \mathrm{\mu M}^{-1} \mathrm{~h}^{-1}$, which is an order of magnitude higher than the diffusional association rate of $N$.A complex and can be explained on basis of smaller size of the ligand compared to native insulin and a smaller binding interface area for the $A . L$ complex compared to the N.A complex. The equilibrium dissociation constant for the $A . L$ complex ( $L$ : BSPOTPE), $K_{\text {A.L }}$, was taken to be $10 \mu \mathrm{M}$, as calculated from the photo luminescence (PL) spectrum for BSPOTPE solution

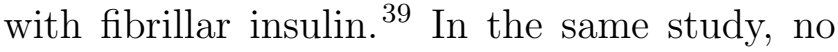
emission was detected for the solution with native insulin, and therefore, our reaction scheme does not involve ligand binding to native insulin. Overall, Scheme $\mathbf{C}$ for ligand inhibition was solved with the schemes with no insulin dimer intermediate with the empirical parameter set (Table 1 Scheme $\mathbf{A}+\mathbf{D}$ or Scheme A only) or the scheme with a dimeric intermediate with parameter set determined in this work (Table 1 Scheme B). The insulin 
concentration was kept fixed at $500 \mu \mathrm{M}$ and BSPOTPE concentration was varied between (0 to 500) $\mu \mathrm{M}$ in accordance with the experimental study. Figures 4, S7)(B) shows that the schemes with no dimeric intermediate completely fail to predict the effect of a ligand with specific affinity for the partially folded species. The inclusion of the intermediate provides very accurate estimate for $t_{\text {nu }}$ over the entire ligand concentration range with nucleation time varying over two orders of magnitude, viz. from a few hours in absence of ligand to more than a week $(175 \mathrm{~h})$ at equimolar ligand concentration. This data clearly indicates the important role of competition between formation of an onpathway dimeric intermediate involving species $A$ leading to aggregation and inhibition of this pathway by a small molecule ligand with high affinity for the hot spot residues of $A$ involved in dimer formation. The $A . L$ complex has a stability comparable to the insulin dimer, viz. $K_{\mathrm{A} . \mathrm{L}}=10 \mu \mathrm{M}$ versus $K_{A_{2}}=(10$ to 45$) \mu \mathrm{M}$, but a significantly higher association rate. Even a one order of magnitude change in $K_{\mathrm{A} . \mathrm{L}}$, at fixed $k_{\mathrm{aL}}$, gives essentially the same $t_{\mathrm{nu}}$ (Figure $\mathrm{S7}(\mathrm{A})$ ), indicating that within the range studied ligand association rate has a more important role than the ligand dissociation rate in prediction of aggregation nucleation time. Further, this implies that free energy estimation methods that provide reasonably accurate estimates for protein-ligand binding free energy 5 can be combined with the approach presented here to provide a fully predictive tool for accurate estimation of ligand effects on protein aggregation inhibition.

To summarize, we have determined a set of transferable parameters for extended LumryEyring aggregation kinetics of insulin using a combination of techniques for modeling elementary events spanning several orders of timescales (milliseconds to hours) while still accounting for specific inter-molecular interactions : insulin folding kinetics from allatomistic metadynamics simulations (prior work), an aggregation pathway dimeric intermediate from structural bioinformatics and multiscale molecular dynamics simulations, and dimer association rates from the transition com- plex theory. Interestingly, we found that association kinetics for kinetically dominant modes for formation of an aggregation-prone insulin dimer have characteristics very similar to that of native protein association such as a transition state at the outer boundary of a deep and narrow bound state ensemble. A single set of physically meaningful kinetic parameters was shown to provide very accurate estimates for changes in aggregation nucleation time from less than a hour to over a week with changes in either insulin or the ligand (BSPOTPE) concentration. We expect these conclusions to be valid in general for other globular proteins. Finally, we note that the approach presented here can be directly applied to quantitatively estimate effect of small molecule ligands on aggregation inhibition of globular proteins, thereby, greatly aiding in development of high stability therapeutic formulations using highthroughput computational screening methods. Development of computational and experimental approaches for determination of kinetics and thermodynamics of non-native association involving slow conformational rearrangement can further the application of kinetic modeling to protein aggregation.

\section{Methods}

\section{Diffusional Association Complex}

The all atomistic (AA) N.A complex was moved perpendicular to the binding interface by a surface separation of $2 \lambda_{b} \quad\left(\lambda_{b}\right.$ : Debye length) and was then converted into MARTINI coarsegrained (CG) representation. The shortranged non-bonded interactions between CG beads were scaled down since earlier studies have shown that the MARTINI forcefield overestimates protein-protein interactions. $\stackrel{57}{5}$ The reduced interaction strength was chosen such that more than half of rigid-body docked N.A dimers, found to be metastable in earlier allatomistic simulations, ${ }^{30}$ stay in an associated state with a stable binding interface during CG simulation (see details in Table S1). CG-MD simulations were carried out using GROMACS 
5.1.4 at aggregation-prone experimental conditions of $338 \mathrm{~K}, \mathrm{pH} 2$, and $600 \mu \mathrm{M}$ insulin. Elastic restraints on backbone of both metastable species $(N \& A)$ were implemented using the ELNEDYNE algorithm. $\frac{58}{\text { The charge state of }}$ the titrable residues (HIS, LYS, ARG) and the end terminals was fixed according to their $p K_{\mathrm{a}}$ (obtained using the propka server ${ }^{\sqrt[59]{9}}$ ). Details of the protocol to obtain a total charge of $+7 e$ on the dimer, in accordance with ion mobility mass spectroscopy measurements, are provided in our previous work. ${ }^{30}$ The final structure from CG-MD simulations was refined by backmapping to the AA representation followed by a $20 \mathrm{~ns}$ MD run using the CHARMM36 forcefield. .60 The central structure of the largest cluster from the stable portion of this AA MD trajectory was taken as the diffusional association complex. The details of simulation parameters, equilibration protocol, and validation of simulation convergence for AA and CG MD simulations are provided in Section S1.A.1 and S1.A.2. The diffusional association rate for a unique set of $N$.A dimers (see Section Linear Integer Programming) was calculated using the transition complex theory ${ }^{28}$ (See S1.3). To this end, we used the Transcomp server ${ }^{33}$ that takes as input the structure of the bound complex and the ionic strength (set to $100 \mathrm{~mm}$ in accordance with simulations and other calculations in this study).

\section{Linear Integer Programming (LIP)}

The obtained N.A dimers were classified into a smallest number of clusters such that structures in the same cluster have similar polar contacts while in different clusters have sufficiently distinct contacts. A set of unique polar contacts at the binding interface was defined using the criterion applied for formation of an encounter complex in Brownian dynamics simulations ${ }^{61}$ (see Figure S4B). The optimization problem to determine the smallest number of clusters with a central structure, referred to as the parent structure, assigned to each cluster was solved using LIP with following set of constraints: (1) any two parent structures should have less than two common polar contacts, (2) any structure with $\geq 2$ common polar contacts with all structures in a cluster was assigned to that cluster. Full details on implementation of LIP are given in Section $\mathbf{S 1 . 2}$

\section{Binding Energy}

Standard-state binding energy for the N.A dimer and the A.L complex, $\Delta G^{\circ}$, was calculated using the implicit-solvent molecular mechanics Poisson-Boltzmann surface area (MMPBSA) method. We have used the single trajectory approach, shown to have smaller standard error than the multiple trajectory approach, wherein averages for both the apo (unbound) and holo (in complex) states are calculated from the MD trajectory of only the solvated complex. ${ }^{62}$ A trajectory of $20 \mathrm{~ns}$ was used for both the complexes. The individual contributions to $\Delta G^{\mathrm{o}}$, as given by equation $\mathrm{S} 8$, were obtained using the g_mmpbsa utility. ${ }^{63}$ We have also added the configurational entropy contribution to $\Delta G^{\mathrm{o}}$, which was calculated using a set of atom-specific, empirically determined weights for the buried and the solvent-accessible area of the atoms at the binding interface of given complex. ${ }^{34}$ The $\Delta G^{\mathrm{o}}$ thus obtained is referred to as MM-PBSA-WSAS binding energy. Specific details on individual terms and the parameter values used in calculation are given in Section S1.4.

\section{Population Balance}

The set of ordinary differential equations (ODEs) for various kinetic schemes (given in Section S3) was solved using MATLABR. The range of parameter values used here resulted in a set of stiff ODEs and accordingly, we used the ode23tb solver ( uses a combination of trapezoidal rule and backward difference formula) with a small time step of $0.2 \mathrm{~h}$. Other stiff equation solvers, such as ode15s and ode23s, lead to large deviations from requirement of total protein mass conservation. For the case of unknown kinetic parameter determination, we used the lsqnonfit function in-combination with ode23tb. Within this the total squared 
error between the target and the predicted monomer species concentration up to the nucleation time $t_{\mathrm{nu}}$ was minimized. MultiStart function was used wherein the minimization problem is solved by starting from multiple start points and its output consists of all local solutions identified ranked from best (lowest objective function value) to worst (highest objective function value).

\section{Ligand Association}

Several conformations for the A.L complex were generated using $H e x$ 8.0.0 $0^{64}$ by rigid-body docking of each of the five stable conformations of BSPOTPE $(L)$ reported in Hong et al. ${ }^{39}$ onto centroids defined by each of the ten residues of $A$ identified as binding hotspot residues in the dominant N.A complex. 1000 unique structures were generated for each set (for given centroid and given ligand conformation) and the most stable A.L complex, as per the scoring function in Hex, for each ligand conformation was selected. The 10 structures thus obtained were solvated at $100 \mathrm{~mm} \mathrm{NaCl}$ and a $20 \mathrm{~ns}$ MD trajectory was generated for each complex. The ligand topology was obtained using the cgenff server. ${ }^{65}$ To get the representative $A . L$ complex for the calculation of association rate, first the central structure of the largest cluster was selected from the stable portion of each MD trajectory. Then the structure with the highest $\cos \Theta^{A . L ; N . A}$ (defined in Equation S13) was used as input to the Transcomp server for calculation of $k_{\mathrm{aL}}$. Additional details in section S1.7.

Acknowledgement This work was funded and supported by the Science \& Engineering Research Board, Department of Science \& Technology, GoI (EMR/2017/004218) and Ministry of Human Resource Development, GoI fellowship. The authors thank IIT Delhi HPC facility for computational resources.

Supporting Information Available. Additional methodology details: AA and CG simulation setup, docking and binding energy calculations, linear integer programming, population balance method and comparison of $t_{\mathrm{nu}}$ calculated through different methods.

\section{References}

(1) Tanford, C. Protein Denaturation. Part A. Characterization of the Denatured State. Advances in Protein Chemistry 1968, 23, 121-217.

(2) Rosenberg, A. S. Effects of protein aggregates: an immunologic perspective. The AAPS journal 2006, 8, E501-E507.

(3) Marshall, K. E.; Marchante, R.; Xue, W.-F.; Serpell, L. C. The relationship between amyloid structure and cytotoxicity. Prion 2014, 8, 192-196.

(4) Fontana, A.; Polverino de Laureto, P.; De Filippis, V.; Scaramella, E.; Zambonin, M. Probing the partly folded states of proteins by limited proteolysis. Folding \& design 1997, 2, R17-26.

(5) Eaton, W. A.; Munoz, V.; Hagen, S. J.; Jas, G. S.; Lapidus, L. J.; Henry, E. R.; Hofrichter, J. Fast kinetics and mechanisms in protein folding. Annual review of biophysics and biomolecular structure 2000, 29, 327-359.

(6) Weiss IV, W. F.; Young, T. M.; Roberts, C. J. Principles, approaches, and challenges for predicting protein aggregation rates and shelf life. Journal of pharmaceutical sciences 2009, 98, 1246-1277.

(7) Lomakin, A.; Teplow, D. B.; Kirschner, D. A.; Benedeki, G. B. Kinetic theory of fibrillogenesis of amyloid $\beta$-protein. Proceedings of the National Academy of Sciences of the United States of America 1997, 94, 7942-7947.

(8) Yoshioka, S.; Aso, Y.; Izutsu, K.; Kojima, S. Is stability prediction possible for protein drugs? Denaturation kinetics of beta-galactosidase in solution. Pharmaceutical research 1994, 11, 1721-5.

(9) Brown, S. Intermediates and the folding of proteins L and G. Protein Science 2004, 13, 958-970.

(10) Tozzini, V. Coarse-grained models for proteins. Current Opinion in Structural Biology 2005, 15, 144-150.

(11) Morris, A. M.; Watzky, M. A.; Finke, R. G. Protein aggregation kinetics, mechanism, and curvefitting: A review of the literature. Biochimica et Biophysica Acta (BBA) - Proteins and Proteomics 2009, 1794, 375-397.

(12) Murray, B.; Rosenthal, J.; Zheng, Z.; Isaacson, D.; Zhu, Y.; Belfort, G. Cosolute Effects on Amyloid Aggregation in a Nondiffusion Limited Regime: Intrinsic Osmolyte Properties and the Volume Exclusion Principle. Langmuir 2015, 31, 4246-4254.

(13) Arosio, P.; Beeg, M.; Nicoud, L.; Morbidelli, M. Time evolution of amyloid fibril length distribution described by a population balance model. Chemical Engineering Science 2012, 78, 21-32.

(14) Arosio, P.; Rima, S.; Lattuada, M.; Morbidelli, M. Population balance modeling of antibodies aggregation kinetics. Journal of Physical Chemistry B 2012, 116, 7066-7075.

(15) Nicoud, L.; Arosio, P.; Sozo, M.; Yates, A.; Norrant, E.; Morbidelli, M. Kinetic analysis of the multistep aggregation mechanism of mono- 
clonal antibodies. Journal of Physical Chemistry $B$ 2014, 118, 10595-10606.

(16) Lee, C. C.; Nayak, A.; Sethuraman, A.; Belfort, G.; McRae, G. J. A three-stage kinetic model of amyloid fibrillation. Biophysical Journal 2007, 92, 3448-3458.

(17) Nielsen, L.; Khurana, R.; Coats, A.; Frokjaer, S.; Brange, J.; Vyas, S.; Uversky, V. N.; Fink, A. L. Effect of Environmental Factors on the Kinetics of Insulin Fibril Formation: Elucidation of the Molecular Mechanism †. 2001,

(18) Dzwolak, W.; Grudzielanek, S.; Smirnovas, V.; Ravindra, R.; Nicolini, C.; Jansen, R.; Loksztejn, A.; Porowski, S.; Winter, R. EthanolPerturbed Amyloidogenic Self-Assembly of Insulin: Looking for Origins of Amyloid Strains $\dagger$. 2005,

(19) Roberts, C. J. Kinetics of irreversible protein aggregation: analysis of extended Lumry- Eyring models and implications for predicting protein shelf life. The Journal of Physical Chemistry B 2003, 10\%, 1194-1207.

(20) Zacharias, M. Accounting for conformational changes during protein-protein docking. Current opinion in structural biology 2010, 20, 180-186.

(21) Seeliger, D.; de Groot, B. L. Conformational Transitions upon Ligand Binding: Holo-Structure Prediction from Apo Conformations. PLoS Computational Biology 2010, 6, e1000634.

(22) Phillip, Y.; Schreiber, G. Formation of protein complexes in crowded environments-from in vitro to in vivo. FEBS letters 2013, 587, 1046-1052.

(23) Nanev, C. N.; Hodzhaoglu, F. V.; Dimitrov, I. L. Kinetics of Insulin Crystal Nucleation, Energy Barrier, and Nucleus Size. Crystal Growth \& Design 2011, 11, 196-202.

(24) Vestergaard, B.; Groenning, M.; Roessle, M.; Kastrup, J. S.; Van De Weert, M.; Flink, J. M.; Frokjaer, S.; Gajhede, M.; Svergun, D. I. A helical structural nucleus is the primary elongating unit of insulin amyloid fibrils. PLoS Biol 2007, 5, e134.

(25) Smoluchowski, M. V. Drei Vortrage uber Diffusion, Brownsche Bewegung und Koagulation von Kolloidteilchen. Zeitschrift fur Physik 1916, 17, 557-585.

(26) Serio, T. R.; Cashikar, A. G.; Kowal, A. S.; Sawicki, G. J.; Moslehi, J. J.; Serpell, L.; Arnsdorf, M. F.; Lindquist, S. L. Nucleated conformational conversion and the replication of conformational information by a prion determinant. Science 2000, 289, 1317-1321.

(27) Singh, R.; Bansal, R.; Rathore, A. S.; Goel, G. Equilibrium Ensembles for Insulin Folding from Bias-Exchange Metadynamics. Biophysical Journal 2017, 112, 1571-1585.

(28) Alsallaq, R.; Zhou, H.-X. Prediction of ProteinProtein Association Rates from a Transition-State Theory. Structure 2007, 15, 215-224.

(29) Harel, M.; Cohen, M.; Schreiber, G. On the dy- namic nature of the transition state for proteinprotein association as determined by doublemutant cycle analysis and simulation. Journal of molecular biology 2007, 371, 180-196.

(30) Mishra, A.; Bansal, R.; Sreenivasan, S.; Dash, R.; Joshi, S.; Singh, R.; Rathore, A. S.; Goel, G. Structure-Based Design of Small Peptide Ligands to Inhibit Early-Stage Protein Aggregation Nucleation. Journal of Chemical Information and Modeling 2020,

(31) Yesylevskyy, S. O.; Schäfer, L. V.; Sengupta, D.; Marrink, S. J. Polarizable water model for the coarse-grained MARTINI force field. PLoS Comput Biol 2010, 6, e1000810.

(32) Gabdoulline, R. R.; Wade, R. C. Protein-protein association: investigation of factors influencing association rates by Brownian dynamics simulations. Journal of molecular biology 2001, 306, 11391155.

(33) Qin, S.; Pang, X.; Zhou, H.-X. Automated prediction of protein association rate constants. Structure (London, England: 1993) 2011, 19, 1744-51.

(34) Wang, J.; Hou, T. Develop and test a solvent accessible surface area-based model in conformational entropy calculations. Journal of Chemical Information and Modeling 2012, 52, 1199-1212.

(35) Strazza, S.; Hunter, R.; Walker, E.; Darnall, D. W. The thermodynamics of bovine and porcine insulin and proinsulin association determined by concentration difference spectroscopy. Archives of Biochemistry and Biophysics 1985, 238, 30-42.

(36) Lindorff-Larsen, K.; Vendruscolo, M.; Paci, E.; Dobson, C. M. Transition states for protein folding have native topologies despite high structural variability. Nature structural $\&$ molecular biology 2004, 11, 443-449.

(37) Best, R. B.; Hummer, G.; Eaton, W. A. Native contacts determine protein folding mechanisms in atomistic simulations. Proceedings of the National Academy of Sciences 2013, 110, 17874-17879.

(38) Librizzi, F.; Rischel, C. The kinetic behavior of insulin fibrillation is determined by heterogeneous nucleation pathways. Protein science 2005, 14, 3129-3134.

(39) Hong, Y.; Meng, L.; Chen, S.; Leung, C. W. T.; Da, L. T.; Faisal, M.; Silva, D. A.; Liu, J.; Lam, J. W. Y.; Huang, X.; Tang, B. Z. Monitoring and inhibition of insulin fibrillation by a small organic fluorogen with aggregation-induced emission characteristics. Journal of the American Chemical Society 2012, 134, 1680-1689.

(40) Yang, Q.-Q.; He, H.; Li, C.-Q.; Luo, L.-B.; Li, S.L.; Xu, Z.-Q.; Jin, J.-C.; Jiang, F.-L.; Liu, Y.; Yang, M. Molecular Mechanisms of the UltraStrong Inhibition Effect of Oxidized Carbon Dots on Human Insulin Fibrillation. Cite This: ACS Appl. Bio Mater 2019, 2020, 217-226.

(41) Gibson, T. J.; Murphy, R. M. Inhibition of insulin fibrillogenesis with targeted peptides. Protein Sci- 
ence 2006, 15, 1133-1141.

(42) Lovatt, M.; Cooper, A.; Camilleri, P. Energetics of cyclodextrin-induced dissociation of insulin. Journal of Inclusion Phenomena and Molecular Recognition in Chemistry 1996, 25, 169-172.

(43) Northrup, S. H.; Erickson, H. P. Kinetics of protein-protein association explained by Brownian dynamics computer simulation. Proceedings of the National Academy of Sciences of the United States of America 1992, 89, 3338-42.

(44) Schlosshauer, M.; Baker, D. Realistic proteinprotein association rates from a simple diffusional model neglecting long-range interactions, free energy barriers, and landscape ruggedness. Protein Sci. 2004, 13, 1660-1669.

(45) Munoz, V.; Thompson, P. A.; Hofrichter, J.; Eaton, W. A. Folding dynamics and mechanism of $\beta$-hairpin formation. Nature 1997, 390, 196-199.

(46) Halford, S. E. Stopped-flow fluorescence studies on saccharide binding to lysozyme. Biochemical Journal 1975, 149, 411-422.

(47) Petty, S. A.; Decatur, S. M. Intersheet rearrangement of polypeptides during nucleation of -sheet aggregates. Proceedings of the National Academy of Sciences 2005, 102, 14272-14277.

(48) Smith, M.; Sharp, J.; Roberts, C. Insulin Fibril Nucleation: The Role of Prefibrillar Aggregates. Biophysical Journal 2008, 95, 3400-3406.

(49) Garai, K.; Frieden, C. The association-dissociation behavior of the ApoE proteins: Kinetic and equilibrium studies. Biochemistry 2010, 49, 95339541.

(50) Khurana, R.; Gillespie, J. R.; Talapatra, A.; Minert, L. J.; Ionescu-Zanetti, C.; Millett, I.; Fink, A. L. Partially folded intermediates as critical precursors of light chain amyloid fibrils and amorphous aggregates. Biochemistry 2001, 40, 3525-3535.

(51) Smith, M. I.; Sharp, J. S.; Roberts, C. J. Nucleation and Growth of Insulin Fibrils in Bulk Solution and at Hydrophobic Polystyrene Surfaces. Biophysical Journal 2007, 93, 2143-2151.

(52) Ahmad, A.; Uversky, V. N.; Hong, D.; Fink, A. L. Early events in the fibrillation of monomeric insulin. The Journal of biological chemistry 2005, 280, 42669-75.

(53) Nettleton, E. J.; Tito, P.; Sunde, M.; Bouchard, M.; Dobson, C. M.; Robinson, C. V. Characterization of the oligomeric states of insulin in self-assembly and amyloid fibril formation by mass spectrometry. Biophysical journal 2000, 79, 1053-1065.

(54) Brange, J.; Andersen, L.; Laursen, E. D.; Meyn, G.; Rasmussen, E. Toward understanding insulin fibrillation. Journal of pharmaceutical sciences 1997, 86, 517-25.

(55) Amdursky, N.; Gazit, E.; Rosenman, G. Formation of low-dimensional crystalline nucleus region during insulin amyloidogenesis process. Bio- chemical and Biophysical Research Communications 2012, 419, 232-237.

(56) Mobley, D. L.; Dill, K. A. Binding of smallmolecule ligands to proteins: "what you see" is not always "what you get". Structure 2009, 17, 489498.

(57) Stark, A. C.; Andrews, C. T.; Elcock, A. H. Toward Optimized Potential Functions for Protein-Protein Interactions in Aqueous Solutions: Osmotic Second Virial Coefficient Calculations Using the MARTINI Coarse-Grained Force Field. Journal of Chemical Theory and Computation 2013, 9, 4176-4185.

(58) De Jong, D. H.; Singh, G.; Bennett, W. F.; Arnarez, C.; Wassenaar, T. A.; Schäfer, L. V.; Periole, X.; Tieleman, D. P.; Marrink, S. J. Improved parameters for the martini coarse-grained protein force field. Journal of Chemical Theory and Computation 2013, 9, 687-697.

(59) Olsson, M. H.; Søndergaard, C. R.; Rostkowski, M.; Jensen, J. H. PROPKA3: consistent treatment of internal and surface residues in empirical p K a predictions. Journal of chemical theory and computation 2011, 7, 525-537.

(60) Vanommeslaeghe, K.; Hatcher, E.; Acharya, C.; Kundu, S.; Zhong, S.; Shim, J.; Darian, E.; Guvench, O.; Lopes, P.; Vorobyov, I.; Mackerell, A. D. CHARMM general force field: A force field for drug-like molecules compatible with the CHARMM all-atom additive biological force fields. Journal of Computational Chemistry 2010, 31, 671-690.

(61) Gabdoulline, R. R.; Wade, R. C. Simulation of the diffusional association of barnase and barstar. Biophysical journal 1997, 72, 1917-29.

(62) Zoete, V.; Meuwly, M.; Karplus, M. Study of the insulin dimerization: Binding free energy calculations and per-residue free energy decomposition. Proteins: Structure, Function and Genetics 2005, 61, 79-93.

(63) Kumari, R.; Kumar, R.; Consortium, O. S. D. D.; Lynn, A. g_mmpbsa A GROMACS tool for highthroughput MM-PBSA calculations. Journal of chemical information and modeling 2014, 54, 1951-1962.

(64) Ritchie, D. W.; Venkatraman, V. Ultra-fast FFT protein docking on graphics processors. Bioinformatics 2010, 26, 2398-2405.

(65) Vanommeslaeghe, K.; MacKerell, A. D. Automation of the CHARMM general force field (CGenFF) I: Bond perception and atom typing. Journal of Chemical Information and Modeling 2012, 52, 3144-3154. 